\title{
CA-125 Predicts Nodal and Distant Metastases in Preoperative Stage I low-Risk Endometrial Cancer
}

L. Nobrega $^{1}$, R. Reis ${ }^{1}$, C. Andrade ${ }^{1}$, R. Schmidt ${ }^{1}$, M. Vieira ${ }^{1}$

${ }^{1}$ Barretos Cancer Hospital, Gynecologic Oncology Department, Barretos, Brazil.

\section{OBJECTIVES}

This study aims to estimate the serum CA-125 concentration as a predictor of nodal and distant metastases in preoperative stage I Endometrial Cancer (EC).

\section{METHODS}

Medical record review was conducted on preoperative imaging-based stage I EC patients who underwent lymphadenectomy between January 2014 and December 2017 in a reference cancer center in Barretos, Brazil. Preoperative CA125 levels were evaluated to quantify the predictive ability of nodal or distant metastases by Mann-Whitney test and receiver operating characteristic (ROC) curve.

\section{RESULTS}

A total of 146 EC cases were included: 104 (71.2\%) stage IA and $42(28.8 \%)$ stage IB with mean age of 61.75 (8.5) years and Body Mass Index (BMI) of $31.6(6.2) \mathrm{kg} / \mathrm{m}^{2}$. Sentinel lymph node mapping was performed in 96 (65.75\%) patients; while 86 (58.9\%), 84 (57.53\%) and 56 $(38.36 \%)$ underwent left, right pelvic and retroperitoneal systematic lymphade-nectomy, respectively. Low-risk EC, comprising endometrioid G1 e G2, was present in 82 (56.2\%) and high-risk disease (endometrioid G3, serous and carcinosarcoma) in 64 (43.8\%). After oncological surgery, nodal and/or distant metastases were found in 18 (12.33\%) cases.

CA-125 levels were higher in low-risk women with pathological evidence of dissemination, compared to non-metastatic disease [24.86 (20.4$56.5)$ vs. $11.4(7.05-17.8) \mathrm{U} / \mathrm{mL}, \boldsymbol{p}<0.001]$, with sensitivity of $85.71 \%$ and specificity of $83.56 \%$, at a concentration threshold of $19.55 \mathrm{U} / \mathrm{mL}$ (AUC=0.86, $\boldsymbol{p}=\mathbf{0 . 0 0 2}$ )(figure 1 ).

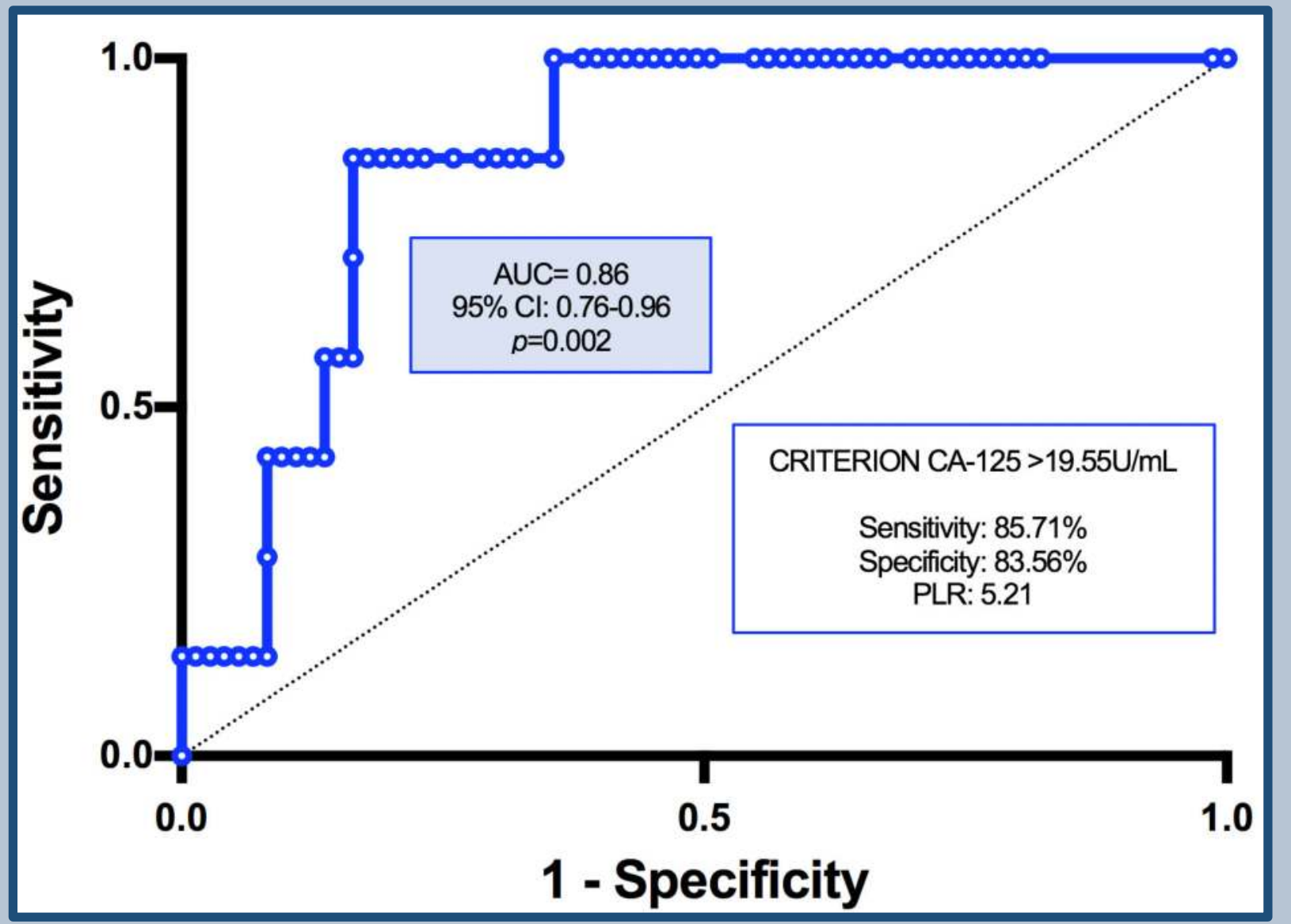

Figure 1: ROC curve for CA-125 prediction of nodal and distant metastases in preoperative stage I lowrisk endometrial cancer

CA-125 values did not attain statistical difference in high-risk patients for prediction of metastasis $(p=0.51)$.

\section{CONCLUSIONS}

CA125 levels are elevated in low-
risk endometrial cancer patients
with nodal and/or distant metastases. Further
studies are warranted to substantiate these
findings and possibly favor CA-125 as a parameter
for an individualized surgical approach.

\section{REFERENCES}

1.Patsner B, Yim GW. Predictive value of preoperative serum CA-125 levels in patients with uterine cancer: The Asian experience 2000 to 2012. Obstet Gynecol Sci. 2013 Sep;56(5):281-8.

2. Takac I, Gorisek B. Serum CA 125 levels and lymph node metastasis in patients with endometrial cancer. Wien Klin Wochenschr. 2006;118 Suppl 2:62-5.

3. Yildiz A, Yetimalar H, Kasap B, Aydin C, Tatar S, Soylu F, Yildiz FS. Preoperative serum CA 125 level in the prediction of the stage of disease in endometrial carcinoma. Eur J Obstet Gynecol Reprod Biol. 2012 Oct;164(2):191-5..

4. Kang S, Todo $Y$, Watari H. Risk assessment of lymph node metastasis before surgery in endometrial cancer: do we need a clinical trial for low-risk patients?. J Obstet Gynaecol Res. 2014 Feb;40(2):322-6. 